\title{
Miniaturized Dual Electromagnetic Oscillatory Actuator for Legged Locomotion of Micro Mobile Robots
}

\author{
Bu Hyun Shin ${ }^{1}$, Kyung-min Lee ${ }^{2}$ and Youngshik Kim ${ }^{1, *}$ \\ ${ }^{1}$ Mechanical Engineering, Hanbat National University, Daejon, 305-719, Korea \\ ${ }^{2}$ Convergence Technology Examination Division 1, Korean Intellectual Property \\ Office \\ youngshik@hanbat.ac.kr
}

\begin{abstract}
In this research we propose a miniaturized dual electromagnetic oscillatory actuator. The proposed actuator is a moving magnet type voice coil actuator with two rotating parts. The structure of the actuator is simple, which includes two magnets, a coil, and a yoke. We provide a linear model to characterize the actuator. We then determine torque and restoring constants using finite element simulation. We also present the dynamic characteristics of the actuator. We propose several types of legged locomotion using dual electromagnetic oscillatory actuators, which is demonstrated and verified using our legged robot prototypes.
\end{abstract}

Keywords: Electromagnetic oscillatory actuator, legged locomotion, micro mobile robot, moving magnet

\section{Introduction}

Recently, many researchers pay attention to micro actuators with growing research interests in micro mobile robots. Thus, many biologically inspired robots similar to insects have been designed. Small creature's mobility can be characterized by several forms of locomotion, which includes legged, earthworm -like, and inchworm-like locomotion. In legged locomotion, some legs contact the ground while the other legs swing. Toward this goal, micro rotary joints should be developed since joints in insects mainly provide rotary motion.

A three-legged miniature robot [1], ambulatory robot HAMR [2], and myriapod robot [3] with legged locomotion were developed using piezo actuators. A piezo actuator is one of popular micro actuators. The piezo actuators provide faster responses and larger forces compared to their size. However, they require higher voltage inputs while producing limited movement. They also need additional mechanisms to transduce linear motion to rotating motion. More recently, polymer actuators are introduced, which includes Ionic Polymer Metal Composite (IPMC) actuators. An eight-legged walking micro robot [4] was also developed using cast IPMC actuators. Applying lower voltage inputs, these actuators provide larger deflection in aqueous solution whereas they do smaller deflection and lower force in air.

Due to their scalability and short response time, electromagnetic voice coil actuators are widely used in miniature devices and optical micro mirrors. Their representative applications include hard-disk drive actuators [5], auto focus actuators for digital cameras [6], and optical micro mirrors actuators [7]. Electromagnetic actuators also provide short response time and proper force. These actuators can be produced massively and inexpensively. DC motors are used in some micro mobile robots [8]. However, it is generally difficult to apply DC motors 
to micro robots since they require additional gear reducers to provide desired force and speed. As a result, the electromagnetic oscillatory actuator for micro mobile robot is developed with a simple structure $[9,10]$. This actuator produces oscillatory motion, which is suitable for various micro mobile robots such as tadpole, 2-legged, and 4-legged robots [9].

In this research we propose a novel miniaturized dual electromagnetic oscillatory actuator, which can be applied to miniature optical devices and micro mobile robots. In general, a rotary mirror in a barcode scanner is driven at $50 \sim 60 \mathrm{~Hz}$ and responses of insects lie within $100 \mathrm{~Hz}$. Thus, our actuator is designed to be sufficiently small and provide faster responses for these applications. We can also change system responses by adjusting an air-gap between a magnet and a coil. Further, we model the actuator, which can be used to predict actuator performance.

\section{Actuator Design and Modeling}

\subsection{Actuator Design}

Figure 1 illustrates the design of our moving magnet type voice coil actuator with two rotating parts. This actuator consists of magnets, a coil, and a yoke similar to a micro mirror system in a barcode scanner. The coil wound around the yoke is fixed by an external support. As shown in Figure 2, magnetic field direction is perpendicular to the direction of coil windings. The direction of electromagnetic forces acting on rotating parts depends on the direction of current.

\subsection{Model}

We model a dual oscillatory electromagnetic actuator based on a typical DC motor model. We propose two types of the actuator, which features the same or opposite magnetic field directions from two magnets as illustrated in Figure. 2. If magnetic field directions of magnets are opposite, Figure 2 (a), force directions are same such that resulting orientation directions of rotating parts are same. In contrast, considering Figure 2 (b) where magnetic field directions of magnets are same, forces directions are opposite such that resulting orientation directions of rotating parts are opposite.

We need to consider two different forces in our actuator unlike a DC motor. The magnetic force between the magnet and yoke generates a restoring torque, which pushes the rotating part into the center position as shown in Figure 2 similar to a spring force. When current flows, electromagnetic force rotates the rotating part in the clockwise or counterclockwise direction depending on current direction. Using the angular acceleration, $\ddot{\theta}$, and the moment of inertia, $J$, in the actuator, we have,

$$
J \ddot{\theta}+T_{\text {magnetic }}=T_{\text {elecromagnetic }}
$$

where each torque is nonlinearly correlated with the rotating magnet and coil structure. However, we may linearize torques for given small rotating angles. Note torque generated by the electromagnetic force in a DC motor is proportional to the magnitude of coil current. We may also assume the restoring torque is proportional to the angular displacement. As a result, torques in (1) can be written by,

$$
T_{\text {magnetic }}=K_{m} \theta \text { and } T_{\text {elecromagnetic }}=K_{t} I
$$




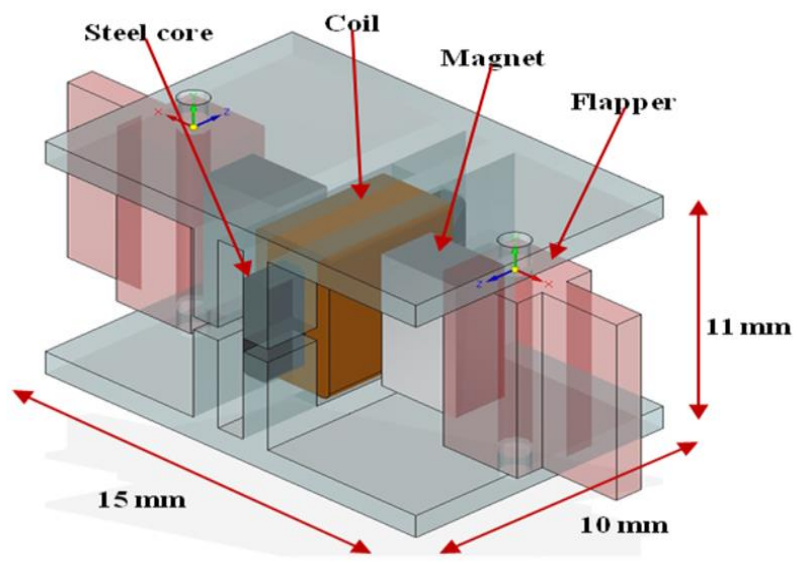

(a) Mechanical Structure of the Actuator

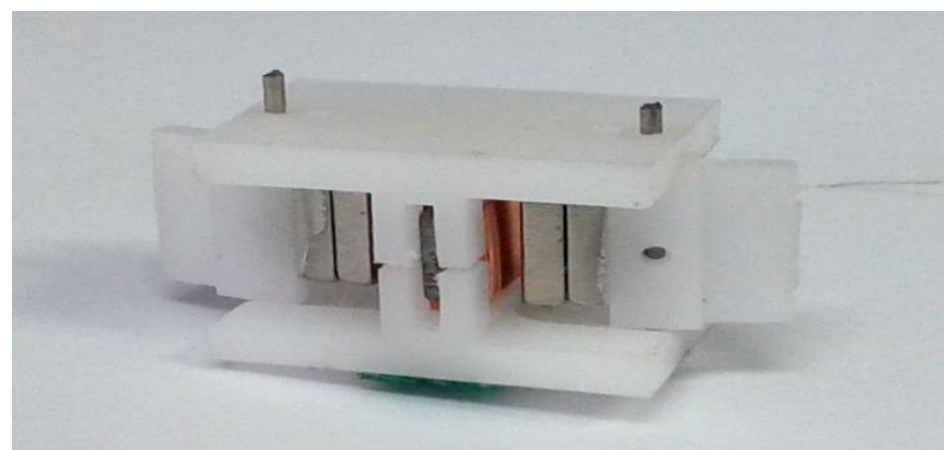

(b) Actuator prototype

\section{Figure 1. Dual Oscillatory Electromagnetic Actuator}

where $K_{t}$ is the torque constant, $K_{m}$ is the restoring constant, $\theta$ is the orientation angle of the rotating part, and $I$ is the magnitude of coil current. Neglecting coil inductance, the circuit equation is,

$$
V=R I+K_{e} \dot{\theta}
$$

where $V$ is the input voltage, $R$ is the coil resistance, and $\dot{\theta}$ is the angular velocity of the rotating part, and $K_{e}$ is the back electromotive force (emf) constant. Note $K_{e}=K_{t}$ in SI units. Using Laplace transforms of (1)-(3), we can derive the second order linear model for our actuator,

$$
\frac{\Theta}{V}=\frac{1}{\frac{J R}{K_{t}} s^{2}+K_{e} s+\frac{K_{m} R}{K_{t}}}
$$




\section{Actuator Design and Modeling}

Using the model (4) derived in Section 2, we can characterize the proposed electromagnetic oscillatory actuator. Toward this goal, we need to find motor parameters. Note the moment of inertia, $J$, and the coil resistance, $R$, are constants given a motor. Measuring the inertia and the resistance from our prototype actuator, we find $J=3.3 \times 10^{-9}$ $\mathrm{kg} / \mathrm{m}^{2}$ and $R=58 \Omega$. Torque and restoring constants are nonlinear parameters. We thus conduct Finite Element Analysis (FEA) to calculate the magnetic force and electromagnetic force. Figure 3 shows magnetic flux density for each Lorentz's force, which is obtained from FEA.

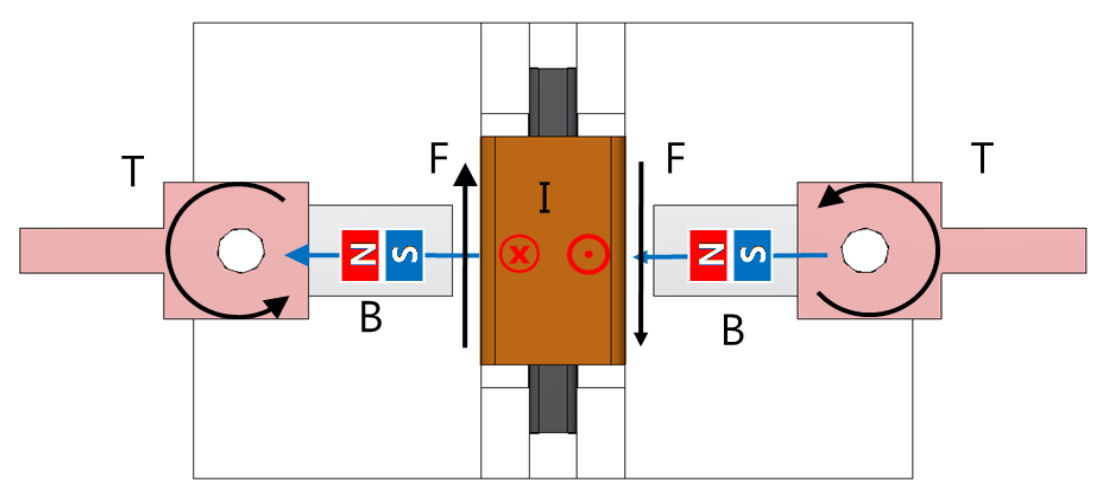

(a) Opposite magnet direction of Lorentz Forces

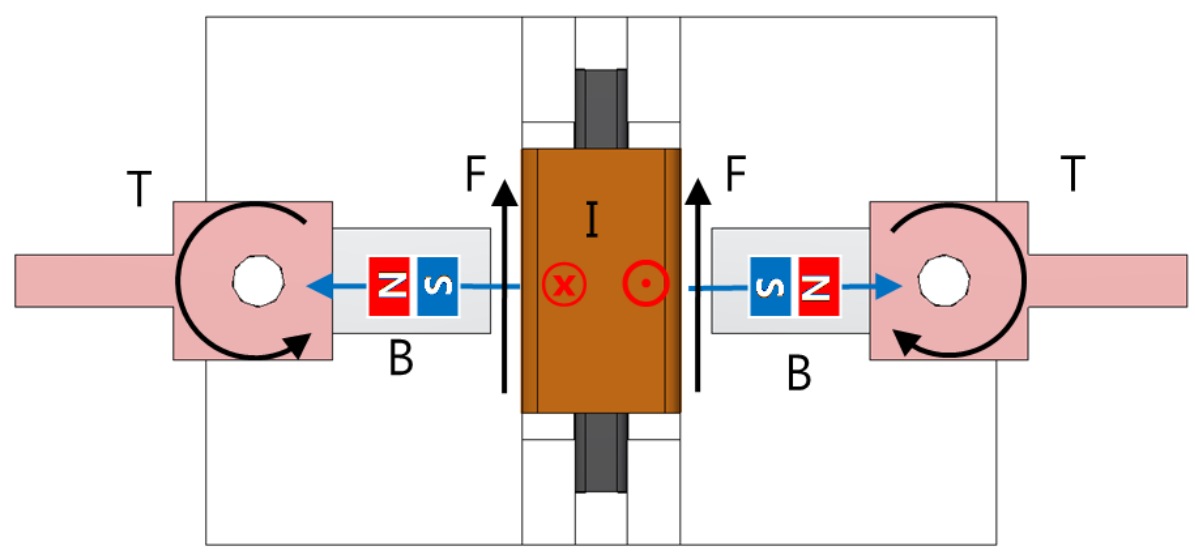

(b) Same magnet direction of Lorentz Forces

Figure 2. Two Type of Actuators and Their Resulting Lorentz Forces 


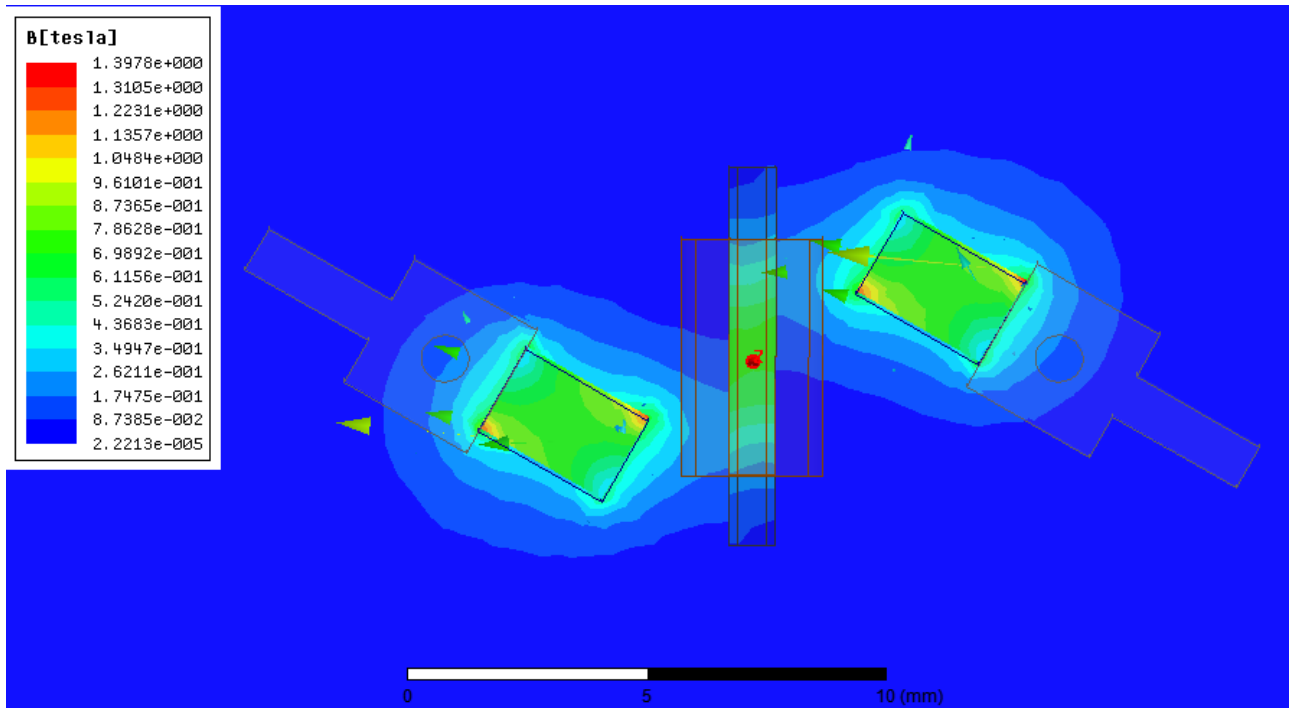

(a) Opposite Magnet Direction

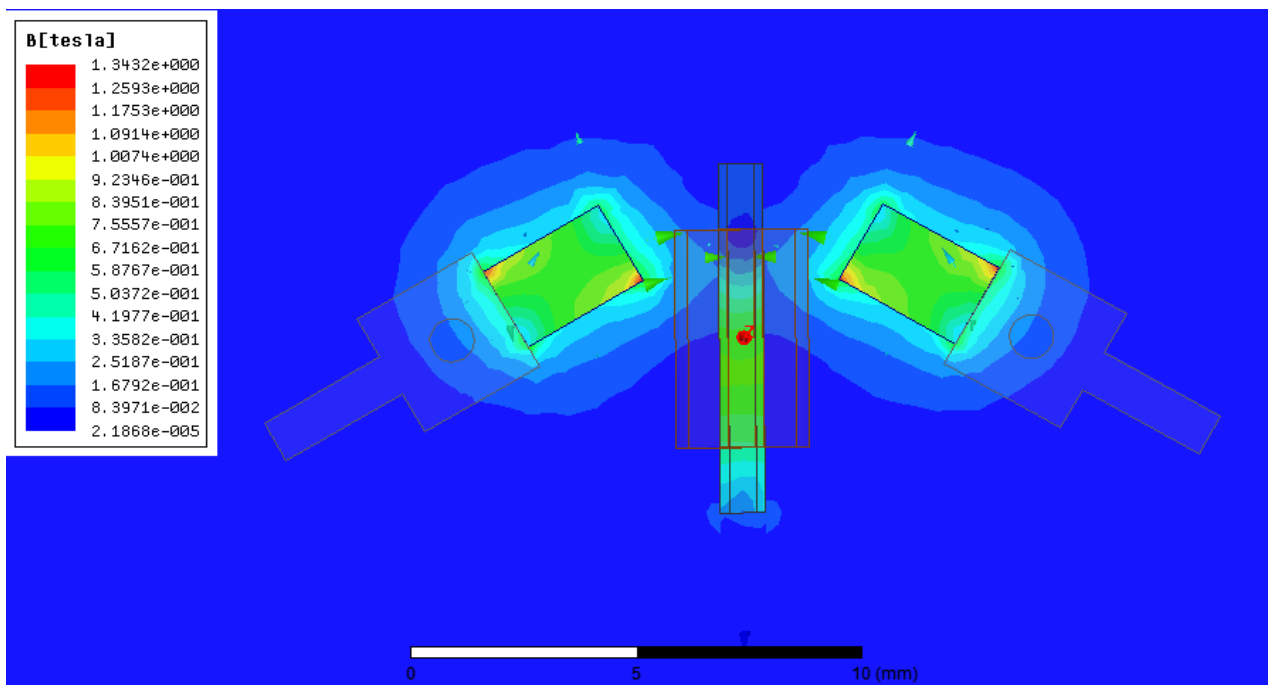

(b) Same Magnet Direction

Figure 3. FEA Results of Magnetic Flux Density

To calculate the restoring constant, we present the restoring torque generated by the magnetic force as a function of the orientation angle of the rotating part in Figure 4, which confirms the restoring torque can be linearized with respect to the orientation angle. As a result, the slope of a linearized line indicates a restoring constant. Similarly, the torque constant can be calculated using linearization of the torque generated by the electromagnetic force with respect to current such that the slope of a linearized line becomes the torque constant as shown in Figure 5. We summarize actuator parameters and constants in Table 1. Applying these properties to the model (4), we can characterize actuator dynamics as shown in Figure 6. 
International Journal of Control and Automation

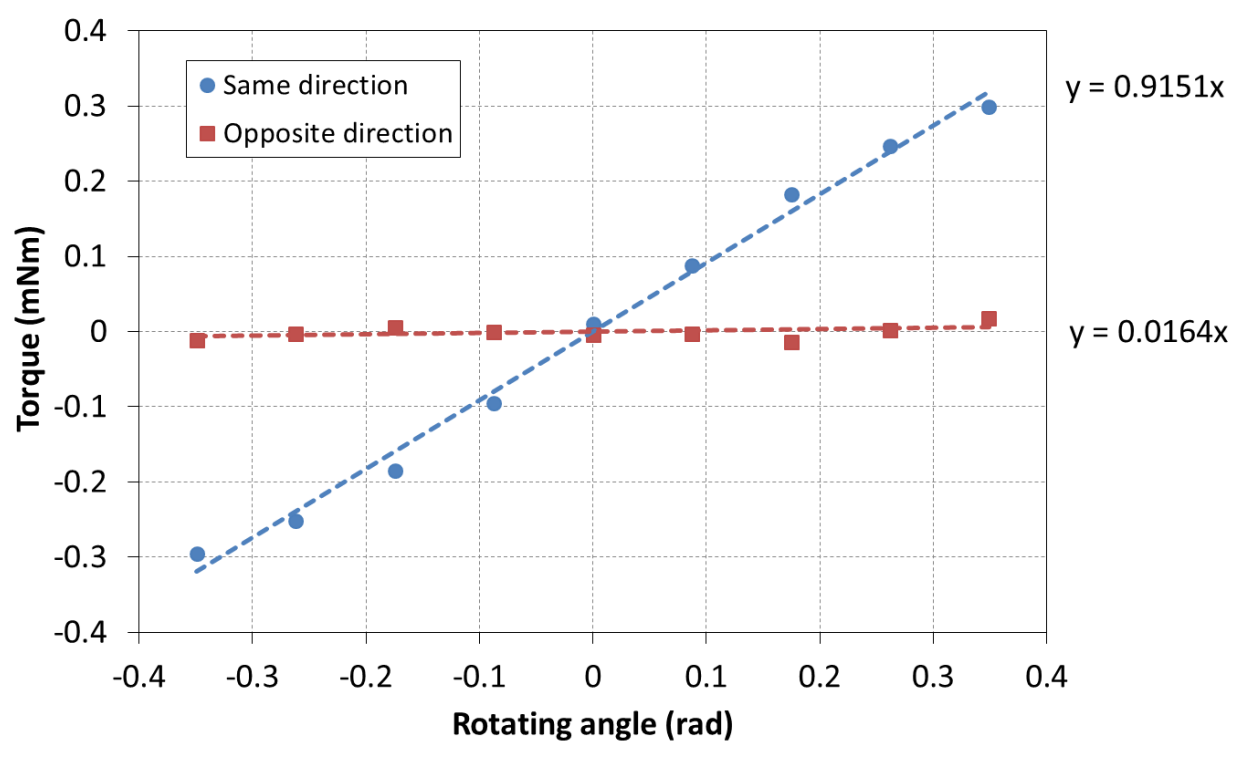

Figure 4. Simulation Result of the Restoring Constant

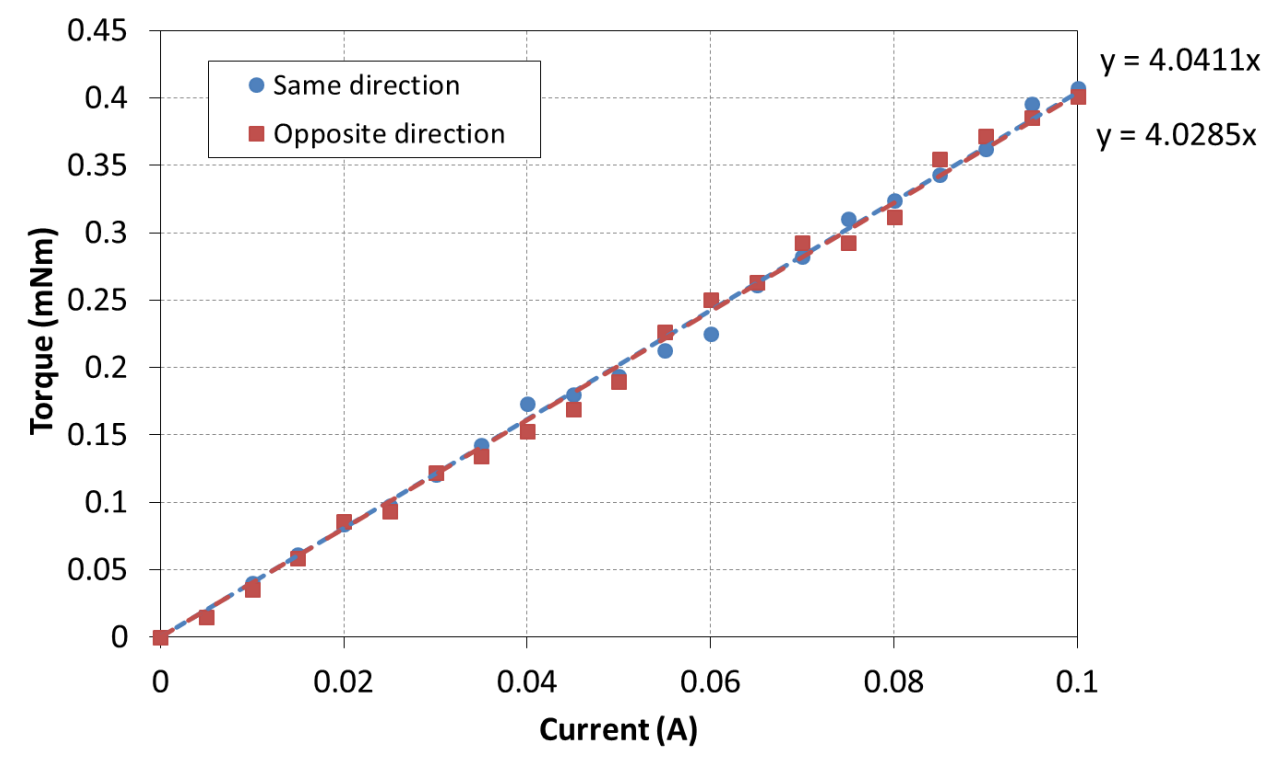

Figure 5. Simulation Result of the Toque Constant 


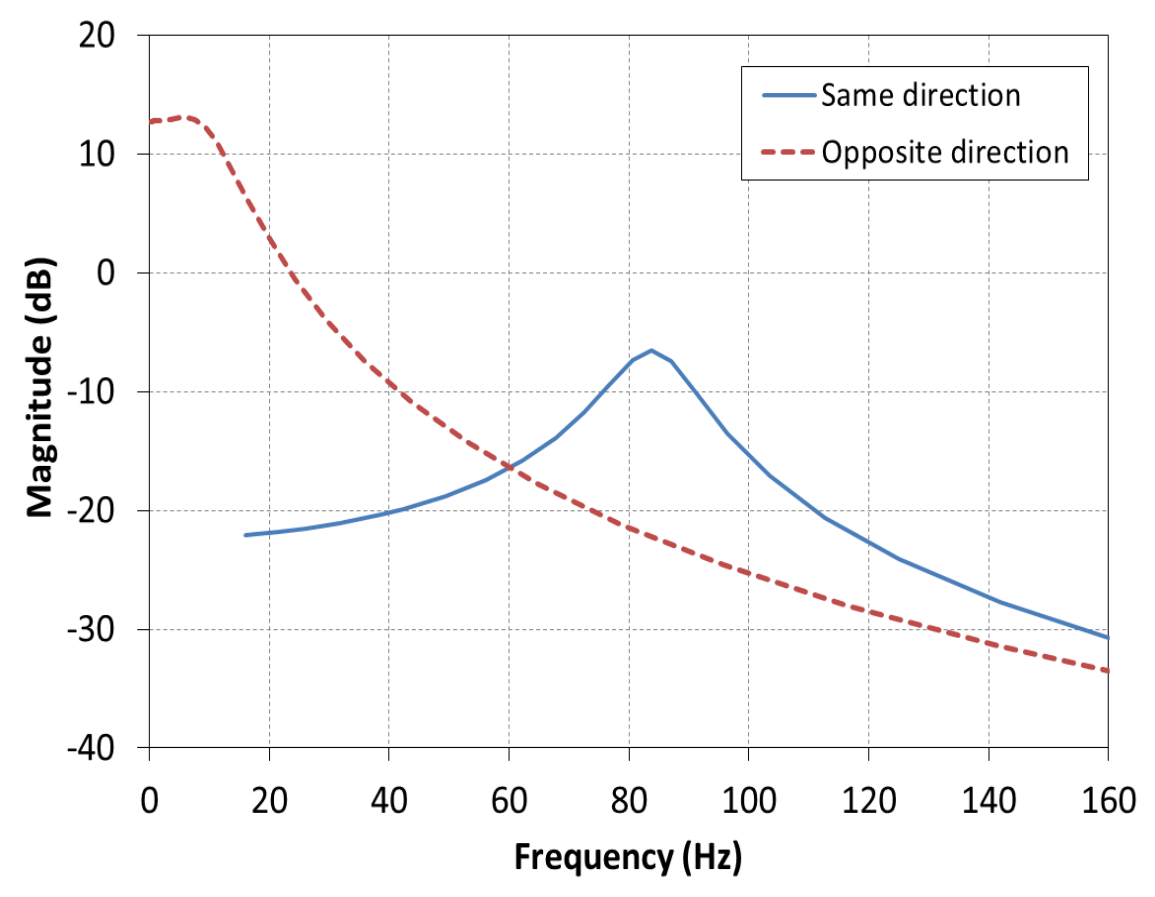

Figure 6. Actuator Dynamic Response Based on the Linear Model

Table 1. Toque Constant and Restoring Constant

\begin{tabular}{ccc}
\hline \hline Parameter & $\begin{array}{c}\text { Magnet } \\
\text { direction }\end{array}$ & Value \\
\hline Inertia $(J)$ & - & $3.3 \times 10^{-9} \mathrm{Kg} / \mathrm{m}^{2}$ \\
\hline Resistor $(R)$ & - & $58 \Omega$ \\
\hline Toque constant $\left(K_{t}\right)$ & Same & $4.04 \mathrm{mNm} / \mathrm{A}$ \\
\cline { 2 - 3 } & Opposite & $4.03 \mathrm{mNm} / \mathrm{A}$ \\
\hline Reinstating constant $\left(K_{m}\right)$ & Same & $0.92 \mathrm{mNm} / \mathrm{rad}$ \\
\cline { 2 - 3 } & Opposite & $0.02 \mathrm{mNm} / \mathrm{rad}$ \\
\hline \hline
\end{tabular}

\section{Legged Locomotion}

\subsection{Quadruped Locomotion}

We propose the quadruped locomotion using two dual electromagnetic oscillatory actuators as shown in Figure 7. Two actuators are configured to have the same direction of the Lorentz force. The leg is made of a steel pin. The moving mechanism of our four-legged robot is based on vibrating slide. For forward motion, the front actuator remains stationary while the rear actuator oscillates back and forth simultaneously as shown Figure 8. The fourlegged robot can provide bi-directional movement depending on which legs are actuated.

The rectangular pulse input signal with amplitude of $\pm 7 \mathrm{~V}$ is applied by using a power opamp driver using a function generator. The proposed quadruped locomotion is experimentally tested using input waves with the frequency of $5 \mathrm{~Hz}$ such that a test result is illustrated in Figure 9. 


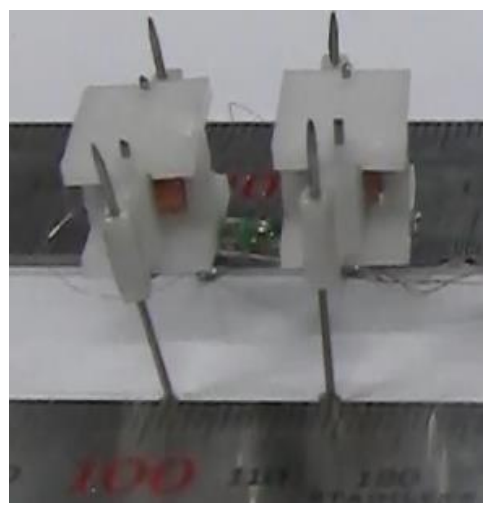

Figure 7. Prototype Robot for Quadruped Locomotion

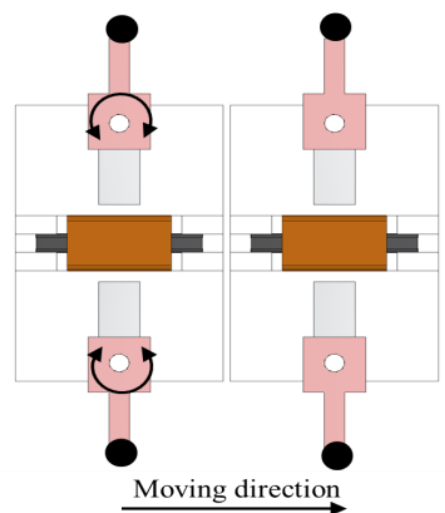

Figure 8. Moving Strategy for Quadruped Locomotion

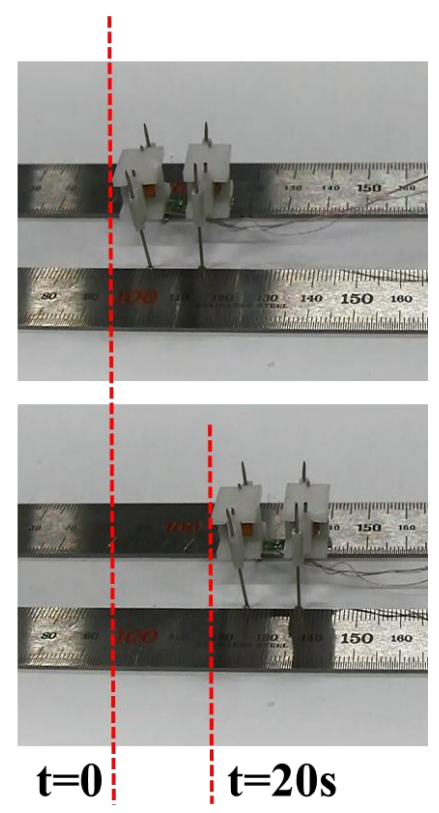

Figure 9. Experimental Locomotion Test Result 


\subsection{Hexapod Locomotion}

We demonstrate the hexapod locomotion using four single electromagnetic oscillatory actuators and a dual electromagnetic oscillatory actuator as shown in Figure 10. In this case, we use the dual electromagnetic oscillatory actuator with the opposite direction of the Lorentz force. The leg is made of ABS plastics. The moving strategy for hexapod robot is relatively well-known as illustrated in Figure 11, which can be easily verified in experiment. Similar to our quadruped robot, the hexapod robot can also provide bi-directional motions simply by changing actuated legs.

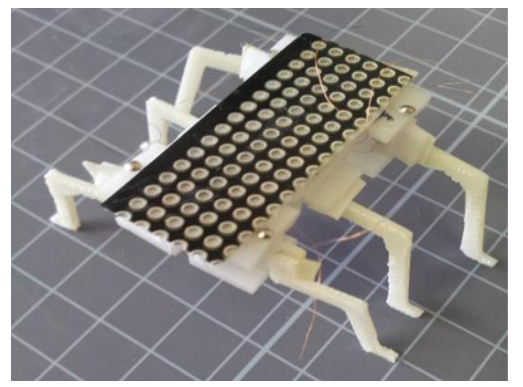

(a) Top view

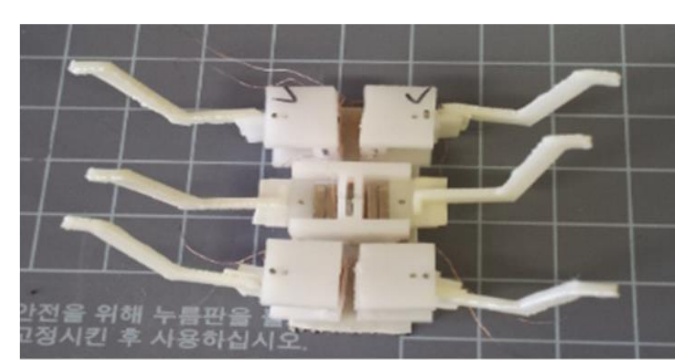

(b) Bottom view

Figure 10. Prototype Robot for Hexapod Locomotion

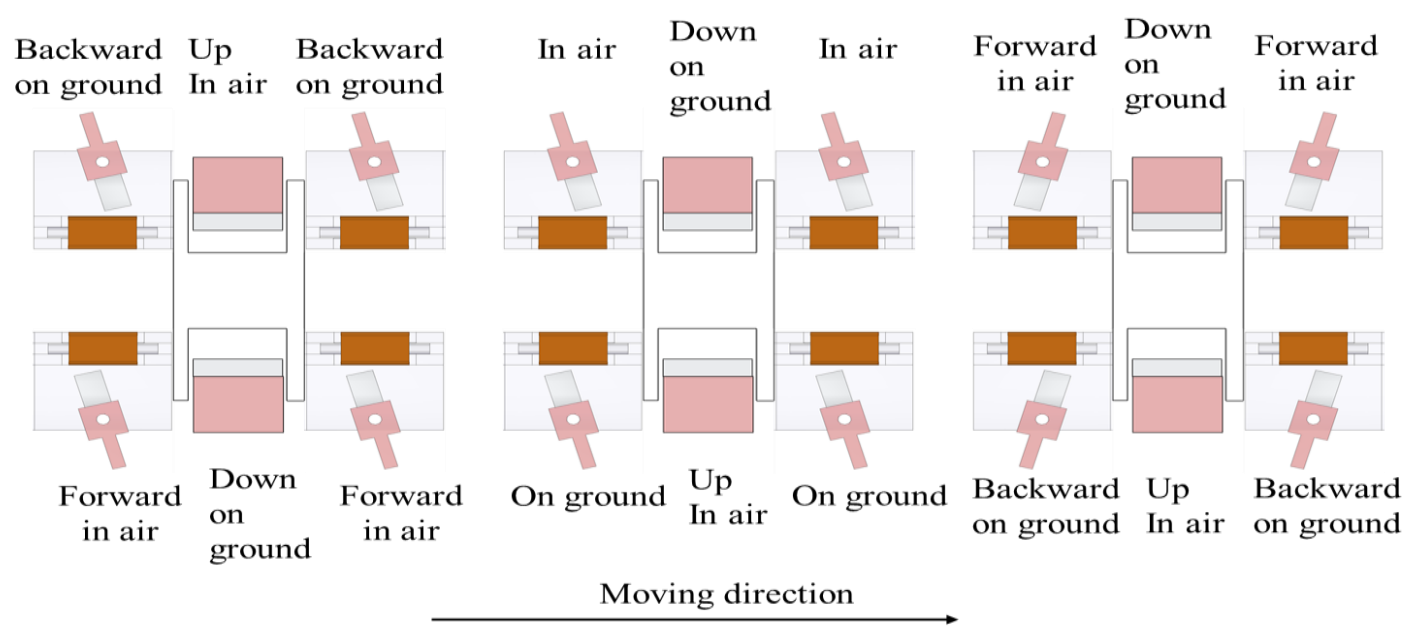

Figure 11. Moving Strategy for Hexapod Locomotion

\subsection{Myriapod Locomotion}

We finally propose myriapod locomotion using four dual electromagnetic oscillatory actuators as shown in Figure 12. Four actuators are configured to have the same direction of the Lorentz force. A leg is made of a steel pin. The moving strategy for myriapod robot is shown Figure 13. In this case, one pair legs slide forward sequentially while the others remain stationary. All legs move then slide backward simultaneously, which drive the robot body to move forward. Similar to the aforementioned locomotion, this myriapod locomotion can also be realized bi-directionally simply by switching actuated legs. Again, the rectangular pulse input signal with amplitude of $\pm 7 \mathrm{~V}$ is used by using a power op-amp driver using a function 
generator. The proposed myriapod locomotion is also tested using input waves with the frequency of $5 \mathrm{~Hz}$ such that an experimental result is shown in Figure 14.

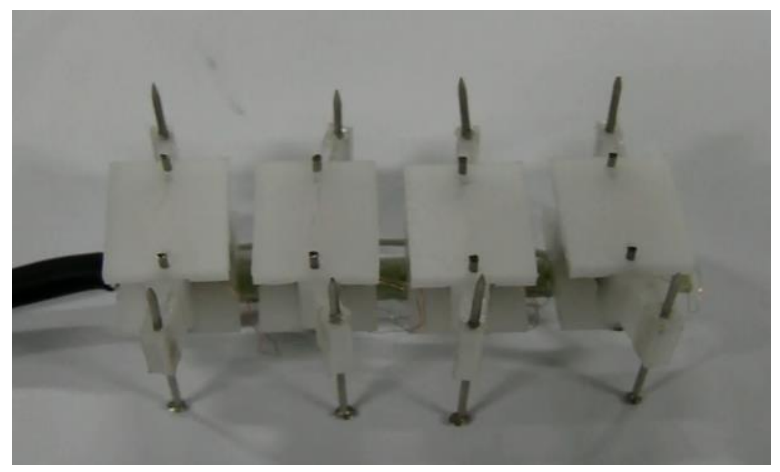

Figure 12. Prototype Robot for Myriapod Locomotion

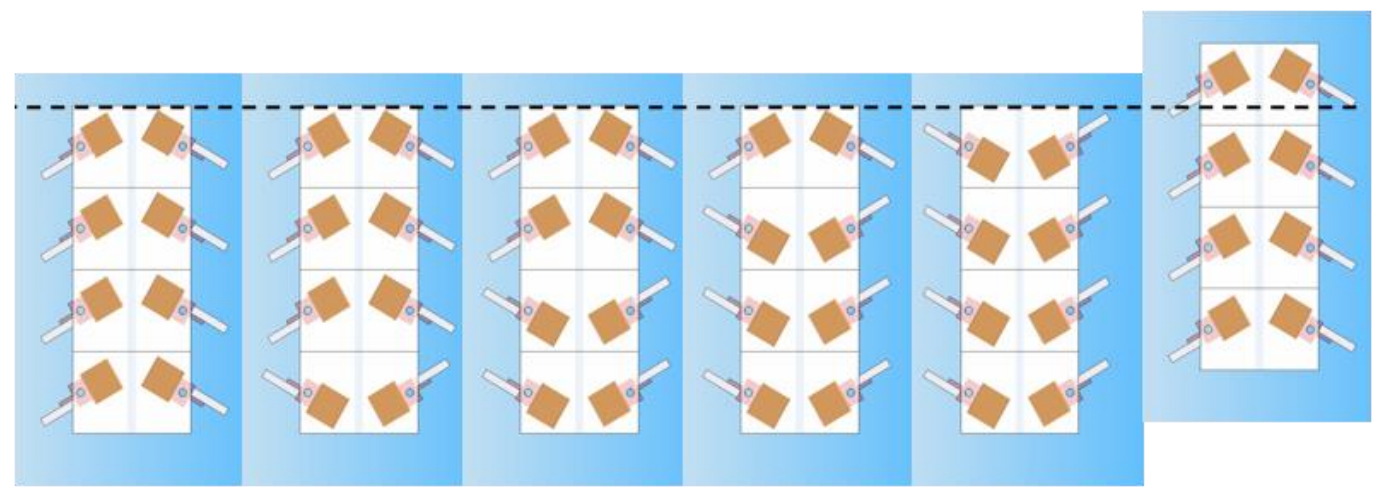

Figure 13. Moving Strategy for Myriapod Locomotion

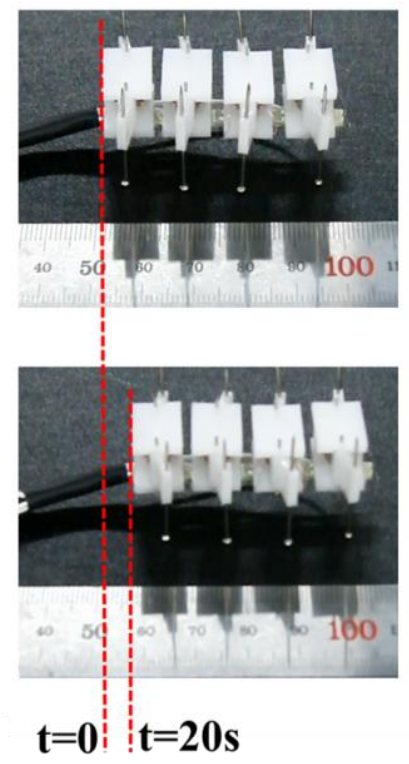

Figure 14. Experimental Locomotion Test Result 


\section{Conclusion}

In this research we propose a novel miniaturized dual electromagnetic oscillatory actuator. The actuator is a moving magnet type voice coil actuator with two rotating parts. We introduce the working principle and linear model for the actuator. We obtain actuator parameters using FEA simulation results. We then present the frequency response of the actuator. Further, we investigate the application of our actuator in several different types of legged locomotion. Our proposed legged locomotion is then verified experimentally using our several different prototype robots with a dual electromagnetic oscillatory actuator. Our experimental results indicate our proposed actuator can be applied to micro mobile and other applications as expected.

\section{Acknowledgements}

This research was supported by Basic Science Research Program through the National Research Foundation of Korea (NRF) funded by the Ministry of Science, ICT \& Future Planning (2012R1A1A1011457). This work was also supported by Business for Cooperative R\&D between Industry, Academy, and Research Institute funded Korea Small and Medium Business Administration in 2013 (Grants No. C0150781).

\section{References}

[1] S. Martel, "Fundamental Principles and Issues of High-speed Piezoactuated Three-legged Motion for Miniature Robots Designed for Nanometer-scale Operations", International Journal of Robotics Research, vol. 7, (2005), pp. 24.

[2] A. T. Baisch, C. Heimlich, M. Karpelson and R. J. Wood, "HAMR3: an autonomous 1.7g ambulatory robot", Proceedings of IEEE International conference of intelligent Robots and System, San Francisco, USA, (2011) September 25-30.

[3] K. L. Hoffman and R. J. Wood, “Autonomous Robots”, vol. 1, (2011), pp. 31.

[4] B. Kim, J. Ryu, Y. Jeong, Y. Tak, B. Kim and J. Park, "A ciliary based 8-Legged walking micro robot using cast IPMC actuators", Proceedings of IEEE International Conference of Robotics and Automation, Taipei, Taiwan, (2003) September 14-19.

[5] B. H. Shin, K.-H. Kim and S.-Y. Lee, "Microsyst”, Technol., vol. 1, (2010), pp. 17.

[6] K.-H. Kim, S.-Y. Lee and S. Kim, "Opt. Express”, vol. 7, (2009), pp. 17.

[7] B. H. Shin, D. Oh and S.-Y. Lee, "Sensors (Basel)", vol. 4, (2013), pp. 13.

[8] D. Lee, S. Kim, Y.-L. Park and R. J. Wood, "Design of centimeter-scale inchworm robots with bidirectional claws", Proceedings of IEEE International Conference of Robotics and Automation, Shanghai, China, (2011) May 9-13.

[9] B. H. Shin and S.-Y. Lee, "Micro mobile robots using electromagnetic oscillatory actuator", Proceedings of the 4th IEEE RAS EMBS International Conference of Biomedical Robotics and Biomechatronics, Rome, Italy, (2012) June 24-27.

[10] B. H. Shin, S.-Y. Lee, K.-M. Lee and D. Oh, “Trans. Korean Soc. Mech. Eng”. A, vol. 12, (2013), pp. 37.

\section{Authors}

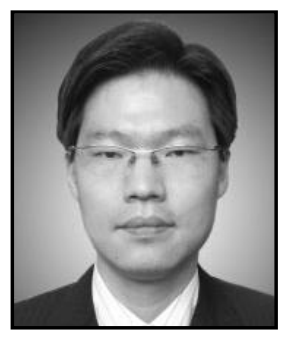

Bu Hyun Shin, received his B.S. and Ph.D. degrees in Mechanical Engineering from Seoul National University, Korea, in 2001 and 2007. $\mathrm{He}$ was a senior engineer in Samsung electronics company, Semiconductor Division from 2007 to 2009. He was a research professor in the Department of Mechanical Engineering at Sogang University, Korea, from 2009 to 2011. He is currently an assistant professor in the Department of Mechanical Engineering at Hanbat National University, Korea. His research interests include actuators, humanoids and biomimetics. 


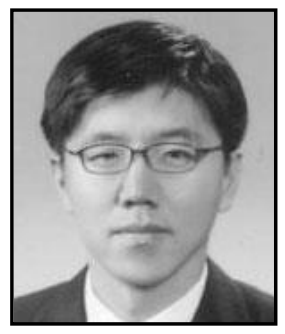

Kyung-min Lee, received his B.S. and Ph.D. degrees in Mechanical Engineering from Seoul National University, Korea, in 2000 and 2006. $\mathrm{He}$ joined the Microcellular Plastics and Manufacturing Lab. at the University of Toronto as a Post-Doctoral Fellow. He carried out researches on robotics at Hyundai Motors Company from 2010 to 2012. $\mathrm{He}$ is currently with the Convergence Technology Examination Division 1, Korean Intellectual Property Office.

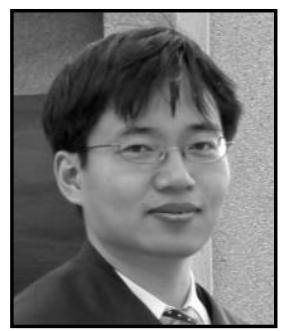

Youngshik Kim, received the B.S. degree from Inha University, Korea in 1996, and the M.S. and Ph.D. degrees from the University of Utah, USA, in 2003 and 2008, respectively, all in mechanical engineering. He is currently an assistant professor in the department of mechanical engineering at Hanbat National University, Korea. His research interests include nonlinear mechanical systems, motion controls, mobile robots, and hybrid mobility for robotic systems. 